\title{
Sex Differences in Retinal Microvasculature Through Puberty In Type 1 Diabetes: Are Girls at Greater Risk of Diabetic Microvascular Complications?
}

\author{
Paul Benitez-Aguirre, ${ }^{1,2}$ Maria E. Craig, ${ }^{1-3}$ Helene G. Cass, ${ }^{4}$ Clare J. Sugden, ${ }^{3}$ Alicia J. Jenkins, ${ }^{5}$ \\ Jie Jin Wang, ${ }^{6,7}$ Janine Cusumano, ${ }^{1}$ Lauren A. B. Hodgson, ${ }^{7}$ Kim Lee, ${ }^{7}$ Tien Yin Wong, ${ }^{6,8}$ \\ and Kim C. Donaghue ${ }^{1,2}$
}

\author{
${ }^{1}$ The Children's Hospital at Westmead, New South Wales, Australia \\ ${ }^{2}$ Discipline of Paediatrics and Child Health, New South Wales, University of Sydney \\ ${ }^{3}$ School of Women's and Children's Health, University of New South Wales, New South Wales, Australia \\ ${ }^{4}$ Department of Ophthalmology, Westmead Hospital, New South Wales, Australia \\ ${ }^{5}$ Clinical Trials Centre, University of Sydney, New South Wales, Australia \\ ${ }^{6}$ Centre for Eye Research Australia, Royal Victorian Eye and Ear Hospital, University of Melbourne, Australia \\ ${ }^{7}$ Centre for Vision Research, University of Sydney, Sydney, Australia \\ ${ }^{8}$ Singapore Eye Research Institute, National University of Singapore, Singapore
}

Correspondence: Kim C. Donaghue, Institute of Endocrinology and Diabetes, The Children's Hospital at Westmead, Locked Bag 4001, Westmead, NSW 2145, Australia; kim.donaghue@health.nsw.gov.au.

Submitted: July 2, 2014

Accepted: November 16, 2014

Citation: Benitez-Aguirre P, Craig ME, Cass HG, et al. Sex differences in retinal microvasculature through puberty in type 1 diabetes: are girls at greater risk of diabetic microvascular complications? Invest Ophthalmol Vis Sci. 2015;56:571-577. DOI:

10.1167/iovs.14-15147
Purpose. Adolescent females with type 1 diabetes (T1D) are reported to have greater risk of early microvascular complications than males. We hypothesize sex differences in retinal vascular geometry (RVG) through puberty are associated with earlier-onset microvascular complications.

Methods. Prepubertal patients ( $n=64,35$ male) with T1D, complication-free at baseline, were followed through to sexual maturity with detailed Tanner-staging and repeated diabetes complications assessments. Retinal vascular geometry from digitized retinal photographs at each visit was assessed using a semiautomated computer program. Determinants of RVG measurements (pre-, during, and post puberty) were explored using generalized estimating equations (GEE). Factors associated with time to onset of retinopathy and albumin excretion rate (AER) were examined using multivariable Cox regression.

Results. Median follow-up was 7.2 years. Retinopathy developed in $69 \%$ and elevated albumin excretion in $56 \%$. In multivariable GEE, female sex was associated with wider venular caliber (prepuberty: lowest-quartile, odds ratio 0.40 [95\% confidence interval: $0.17,0.96]) ; P=0.04$ ) and lower arteriolar length-to-diameter-ratio (LDRa) (during puberty: lowest-quartile 2.87 $[1.01,8.13] ; P=0.047$ and post puberty: $2.93[0.96,8.64] ; P=0.06)$. In Cox-regression, females developed retinopathy earlier than males $(8.1$ vs. 9.6 years; $P=0.002)$. Female sex (hazard ratio [HR] 3.8 [1.6-8.6]; $P=0.002)$ and growth velocity (1.3 [1.1-1.5]; $P=0.001)$ were associated with earlier retinopathy.

Conclusions. This is the first longitudinal study to repeatedly examine RVG through puberty in youth with T1D. Sex dimorphism was observed. Female sex was associated with lower LDRa, wider venules, and earlier onset of retinopathy. These RVG patterns have been associated with incident microvascular complications but did not reach statistical significance in this study. Larger studies are needed to investigate the RVG, microvascular complications, and sex associations early in the course of T1D.

Keywords: retinal vascular geometry, diabetic retinopathy, adolescents, type 1 diabetes, microvascular complications
$\mathrm{P}$ uberty is a high-risk period for type 1 diabetes (T1D) during which microvascular complications appear to accelerate. ${ }^{1,2}$ There is an observed sex dimorphism with younger females being at greater risk for microvascular complications early in the course of diabetes ${ }^{3,4}$ and males developing greater risk later in life. ${ }^{5}$ We previously described a greater risk of incident retinopathy, ${ }^{3}$ while the Oxford Regional Prospective Study (ORPS) demonstrated a greater risk for microalbuminuria, in adolescent females with T1D. ${ }^{4}$
Retinal vascular geometry is a predictor of future complications in adolescents with T1D.,7 Little is known, however, about the retinal microvascular geometry during puberty and there are no longitudinal data examining retinal vascular geometry (RVG) through this critical period. Imaging the retina offers a unique opportunity to noninvasively and repeatedly examine the microvasculature in-vivo, and computer-assisted retinal image analysis has allowed a better understanding of retinal vascular parameters and their relationship with diabetic 
complications in the retina (i.e., retinopathy) and elsewhere (e.g., nephropathy, coronary heart disease). We hypothesized that sex differences in microvascular architecture are associated with earlier onset of microvascular complications in females during puberty. The aim of this study was to longitudinally describe the retinal microvasculature in a cohort of carefully characterized youth with T1D followed from prepuberty through to sexual maturity, to explore sex differences, and associations with retinopathy and early renal dysfunction.

\section{MeTHODS}

\section{Study Population}

We conducted a prospective longitudinal observational study of 64 (35 male, 55\%) patients diagnosed with T1D prior to the onset of gonadarche (Tanner stage B1 in females or testicular volume of $<4 \mathrm{~mL}$ in males) and free of microvascular complications at baseline assessment. Participants were from a previously described cohort of 68 (36 male) participants; three had relocated (two interstate, one overseas) and one was excluded due to microalbuminuria (MA) at baseline. ${ }^{8}$ Median (IQR) prepubertal age was $9.8(9.1-10.4)$ years in girls and 10.7 $(10.2,11.3)$ years in boys $(P<0.001)$. At study closure, there were no sex differences in diabetes duration (female versus male: 9.2 [7.6-11.0] vs. $10.0[7.6-11.0]$ years, $P=0.2$ ) or follow-up duration $(7.4[6.5-7.9]$ vs. $6.9[5.4-7.7]$ years, $P=$ $0.2)$.

All 64 participants underwent repeated retinal fundal photography suitable for retinal vascular geometry assessment. Participants were prospectively followed through to sexual maturity using standardized interviews, clinical examinations, and laboratory investigations at each visit as previously described. ${ }^{8}$ Blood pressure (BP) was measured in the seated position after 5 minutes of rest with a sphygmomanometer using an appropriately sized cuff. Anthropometry was recorded at each visit and annualized growth velocity calculated for each participant. Venous blood was obtained for measurement of HbA1c and total cholesterol levels. HbA1c was measured at each assessment at a central laboratory using the Bio-Rad Diamat analyzer (Bio-Rad, Hercules, CA, USA). Clinical assessments were performed between 1995 and 2004 at the Royal Alexandra Hospital for Children. Written informed consent was obtained from all participants and their guardians. The study was approved by the Royal Alexandra Hospital for Children Ethics Committee.

\section{Definition of Retinopathy}

Mydriatic 7-field stereoscopic fundal photography of both eyes was performed using a Topcon Fundus Camera (TRC 50-VT; Tokyo Optical, Tokyo, Japan) using film. Retinopathy was assessed and graded from these images by a single ophthalmologist masked to participants' clinical characteristics, according to the Early Treatment Diabetic Retinopathy Study (ETDRS) adaptation of the modified Airlie House classification. ${ }^{9}$ Incident Retinopathy was defined as at least one microaneurysm/hemorrhage in either eye (ETDRS level 21, minimal nonproliferative diabetic retinopathy or greater). ${ }^{10,11}$

\section{Definition of Renal Dysfunction}

The mean urinary albumin excretion rate (AER) was calculated from three consecutive timed overnight urine collections. Urinary albumin was measured using a polyclonal radioimmunoassay (Pharmacia AB, Uppsala, Sweden). Early elevation of AER was defined as a mean AER greater than $7.5 \mu \mathrm{g} / \mathrm{min}^{12}$ and microalbuminuria was defined as an AER greater than $20 \mu \mathrm{g} /$ min in two of three timed overnight urine samples or an albumin/Creatinine ratio greater than $2.5 \mathrm{mg} / \mathrm{mM}$ in two of three early morning urine sample collections.

\section{Retinal Vascular Geometry}

All available central retinal film images were individually scanned in a high-resolution scanner $(3071 \times 2048$ pixels $)$ in raw format to avoid pixel and color compression (CanoScan FS2710; Canon, Tokyo, Japan). Digitized images were used to assess retinal vascular geometry. A single grader (LH) individually ensured each image was appropriate for retinal vascular geometry grading. Images were considered to be of poor quality and not gradable if they were blurred or contained incomplete representation of all relevant zones, or if there were fewer than four large arterioles or venules gradable in one image. Deidentified digital retinal images for each participant were independently graded by the single grader (KL) masked to any anthropometric or clinical data at an off-site facility, using the semiautomated, computer-assisted image program Singapore I Vessel Assessment (SIVA) as previously described. ${ }^{7,13,14}$ The grader ensured that the vessel type selected by the program was correct. The software then combined the individual measurements into summary indices:

- Length to diameter ratio of arterioles and venules (LDRa and LDRv, respectively) was calculated as the length from the midpoint of the first branch to the midpoint of the second branch, divided by the diameter of the parent vessel at the first branch.

- Tortuosity is a measure of vessel shape and undulation: Simple tortuosity of arterioles and venules (STa and STv respectively) was calculated as the ratio between the actual path length of the vessel segment (measured by tracking) and the straight-line length of the same segment.

Curvature tortuosity of arterioles and venules (CTa and CTv respectively): Because simple tortuosity does not distinguish between increased length because of bowing and that because of multiple points of inflection, another distinct measure of tortuosity based on vessel curvature was derived from the integral of the square of curvature along the path of the vessel, normalized by the total path length. ${ }^{15}$

- Retinal vessel caliber measurements, represented by the central retinal arteriolar equivalent (CRAE) and the central retinal venular equivalent (CRVE) summarize the caliber of the biggest six arterioles or venules, respectively. ${ }^{16}$

\section{Statistical Analysis}

Data from a total 573 visits were allocated to three pubertal categories for analysis: prepuberty (Tanner stage 1; 50\% male; 268 visits) during-puberty (Tanner stage 2-4; 58\% male, 164 visits) and post puberty (Tanner stage 5 or cessation of linear growth; 52\% male, 141 visits). Descriptive statistics are presented as mean and standard deviation (SD) for normally distributed data or median and interquartile range [IQR] for skewed data. Differences in clinical characteristics between males and females at each puberty stage (pre-, during, and post puberty) were evaluated using Student's $t$-test for normally distributed data, or the Mann-Whitney $U$ test for skewed data (Table 1).

The log-rank test was used to compare median time to onset of complications between males and females. We used Cox proportional hazards regression to examine factors associated 
with time to incident retinopathy and early renal dysfunction (AER $>7.5 \mu \mathrm{g} / \mathrm{min}$ ) in all participants. Covariates analyzed were sex, RVG parameters, growth velocity, systolic blood pressure (SBP) standard deviation scores (SDS), diastolic blood pressure (DBP) SDS, HbA1c, and total cholesterol. Multivariable models were constructed using significant variables from univariable models. Statistical procedures were performed using SPSS version 22.0 (2013; SPSS, Inc., Chicago, IL, USA).

Longitudinal analysis was performed using generalized estimating equations (GEEs) with retinal vascular geometry parameters as outcome measures (Tables 2, 3). Baseline STa, STv, LDRa and LDRv were $\log$ transformed for analysis as continuous variables and categorized into quartiles to examine for threshold effects. Binary outcomes for geometry were used with the adverse quartile compared with the other three quartiles. Explanatory variables examined included sex, puberty, diabetes duration, growth velocity, HbA1c, systolic and diastolic BP SD scores (SBP SDS and DBP SDS), body mass index (BMI) SDS, total cholesterol, AER expressed as log AER, and relevant interaction terms. The use of GEE allowed all visits to be included in the analysis and accounted for correlations between repeated observations for a given patient (Tables 2,3 ).

\section{Results}

All 64 participants were prepubertal at baseline. At the final study visit 51 (26 male) had reached Tanner stage 5; nine (6 male) Tanner stage 4 and three Tanner stage 2 . Median followup period was $7.2(6.3-7.8)$ years with median 9.0 (7.3-10.8) visits per participant. Females had significantly higher cholesterol than males during and post puberty with a trend toward poorer glycemic control and had developed higher BMI SDS and lower DBP SDS than males post puberty (Table 1). Females were younger with shorter duration in all pubertal phases. There was an observed inverted "U-shaped" trend with respect to HbA1c in all participants with better glycemic control apparent pre- and post puberty.

Retinopathy developed in 69\% (44/64) of participants with similar proportions of females and males $\left(72 \%\right.$ vs. $66 \% ; \chi^{2}=$ 0.6). Females had earlier onset retinopathy: median duration 8.1 vs. 9.6 years $(\log \operatorname{rank} P=0.001)$. Of those that developed retinopathy, 39/44 had grade 21 and 5/44 (3 females) had grade 31 retinopathy. Albumin excretion rate greater than $7.5 \mu \mathrm{g} / \mathrm{min}$ developed in $56 \%$ of participants with time to onset 10.1 vs. 12.1 years, $P=0.3$; females vs. males, respectively.

\section{Complications: Cox Regression Analysis}

In multivariable Cox regression analysis, earlier risk of incident retinopathy was associated with female sex (hazard ratio [HR] $2.7[1.3,5.3] ; P=0.002)$ and greater growth velocity (HR 1.3 $[1.1,1.5] ; P=0.001$; Fig.).

Sex was not significantly associated with risk of incident renal dysfunction. Growth velocity was significantly associated with incident renal dysfunction (HR $1.3[1.1,1.5] ; P<0.001$ ) after accounting for HbA1c and cholesterol but not sex.

Retinal vascular geometry was not significantly associated with earlier risk of retinopathy or renal dysfunction (results not shown)

\section{Retinal Vascular Geometry Measures by Pubertal Stage (Table 2)}

Females had wider arterioles (CRAE) and venules (CRVE) than males with sex differences being most notable prepuberty. Length to diameter ratio of arterioles was consistently lower in females through all puberty stages. Females had greater 
arteriolar tortuosity (STa and CTa) and lower venular tortuosity (STv and CTv) with the most significant differences present in the prepuberty period.

\section{Retinal Vascular Geometry: GEE Longitudinal Analysis (Table 3)}

Female sex was associated with wider venules (CRVE upper quartiles: $P=0.04$ ) before puberty and earlier arteriolar branching (LDRa lowest quartile: $P=0.047$ ) during puberty.

There was a trend for females to have greater arteriolar tortuosity (CTa upper quartile: $P=0.08$ ) and lower venular tortuosity (STv and CTv lower quartiles: $P=0.08$ ) prepuberty.

\section{Discussion}

To our knowledge, this is the first prospective longitudinal description of retinal microvascular geometry through puberty; moreover, this is the first time such measures are systematically correlated with clinical phenotypes through puberty in youth with T1D. We observed significant sex differences in retinal microvascular geometry with females having wider venules and arterioles, more complex retinal arteriolar and simpler venular networks, than males starting prepuberty and continuing during puberty. We confirmed that incident diabetic complications occurred earlier in females, which may be underpinned by inherent sex differences in RVG.

Females have more complex retinal arteriolar RVG (lower LDRa and greater STa) and simpler venular networks (lower STv) than prepubescent males. Both these patterns have been previously associated with greater risk of retinopathy and early renal dysfunction respectively. ${ }^{7,13}$ In this cohort, we confirmed that females were at greater risk of developing incident retinopathy earlier than males through puberty. We previously found in a larger cohort of older adolescents with T1D that females were at significantly greater risk of retinopathy than male counterparts. ${ }^{3}$ Similarly, the ORPS reported that female sex was associated with greater risk of microalbuminuria. ${ }^{4}$ In our cohort, we observed a similar trend with respect to early renal dysfunction but did not reach statistical significance.

We observed a sexual dimorphism in RVG in our study cohort consistent with studies performed in nondiabetic ${ }^{17,18}$ and diabetic adult populations ${ }^{19-21}$ studying retinal vascular calibers. The fact that these differences are observed throughout life may reflect the observed sex differences in cardiovascular risk, which are modified by diabetes. Sex specific differences in cardiovascular disease risk are well recognized, ${ }^{22}$ and have been hypothesized to be related to sex differences in microvascular architecture. ${ }^{23-25}$ Premenopausal females have a protective advantage with respect to cardiovascular disease risk compared with male counterparts, which is lost and even exacerbated in females with T1D. ${ }^{22,26}$ Our results consistently demonstrated higher-risk retinal vascular geometry and metabolic profiles (higher HbA1c and cholesterol levels) in females. Interestingly, females demonstrated a narrowing of arterioles during puberty and narrower arterioles have been associated with increased cardiovascular risk. ${ }^{27,28}$ A better understanding of the retinal microvasculature early in the course of diabetes may yield insights to the hemodynamic changes and mechanisms underpinning subsequent micro- and macrovascular complications.

In addition to female sex, higher growth velocity predicted retinopathy (Fig.), paralleling progression through puberty and corresponding linear growth acceleration. The diabetes milieu has adverse metabolic effects on the vasculature and can influence normal pubertal development. ${ }^{29}$ Insulin-like growth 
TABLE 3. Factors Associated with Retinal Vascular Outcomes Stratified by Pubertal Stage-Longitudinal Analysis Using GEE

\begin{tabular}{|c|c|c|c|c|c|c|}
\hline & $\begin{array}{l}\text { Prepuberty } \\
\text { OR }(95 \% \text { CI) }\end{array}$ & $\boldsymbol{P}$ & $\begin{array}{c}\text { During Puberty } \\
\text { OR }(95 \% \text { CI) }\end{array}$ & $\boldsymbol{P}$ & $\begin{array}{l}\text { Post Puberty } \\
\text { OR }(95 \% \text { CI) }\end{array}$ & $\boldsymbol{P}$ \\
\hline \multicolumn{7}{|c|}{ CRAE, fourth vs. other quartiles } \\
\hline Sex, F & $1.79(0.87,3.67)$ & 0.11 & $0.63(0.24,1.64)$ & 0.35 & $1.39(0.50,3.86)$ & 0.53 \\
\hline HbA1c & $1.18(0.89,1.58)$ & 0.25 & $0.68(0.45,1.02)$ & 0.06 & $0.60(0.38,0.95)$ & 0.03 \\
\hline \multicolumn{7}{|c|}{ CRVE, first vs. other quartiles } \\
\hline Sex, F & $0.40(0.17,0.96)$ & 0.04 & $1.75(0.62,4.94)$ & 0.29 & $1.69(0.52,5.45)$ & 0.38 \\
\hline SBP SDS & $1.17(0.79,1.75)$ & 0.43 & $1.90(1.08,3.34)$ & 0.03 & $1.28(0.82,2.01)$ & 0.27 \\
\hline \multicolumn{7}{|c|}{ LDRa, first vs. other quartiles } \\
\hline Sex, $F$ & $2.07(0.77,5.63)$ & 0.14 & $2.87(1.01,8.13)$ & 0.047 & $2.93(0.96,8.94)$ & 0.06 \\
\hline Cholesterol, mM/L & $0.97(0.61,1.52)$ & 0.88 & $1.36(1.01,1.81)$ & 0.04 & $1.31(1.02,1.68)$ & 0.03 \\
\hline \multicolumn{7}{|c|}{ LDRv, fourth vs. other quartiles } \\
\hline Sex, F & $1.16(0.40,3.43)$ & 0.78 & $1.64(0.52,5.20)$ & 0.40 & $0.58(0.18,1.93)$ & 0.38 \\
\hline \multicolumn{7}{|c|}{ STa $\left(\times 10^{3}\right)$, fourth vs. other quartiles } \\
\hline Sex, F & $2.23(0.82,6.05)$ & 0.17 & $0.26(0.44,3.65)$ & 0.67 & $1.13(0.38,3.35)$ & 0.82 \\
\hline \multicolumn{7}{|c|}{ STv, first vs. other quartiles } \\
\hline Sex, F & $2.17(0.92,5.12)$ & 0.08 & $1.42(0.57,3.60)$ & 0.45 & $1.21(0.54,2.73)$ & 0.65 \\
\hline Cholesterol, mM/L & $1.83(0.79,3.66)$ & 0.09 & $2.58(1.45,4.58)$ & 0.001 & $1.55(1.01,2.38)$ & 0.05 \\
\hline DBP SDS & $0.9(0.65,1.24)$ & 0.52 & $0.72(0.45,1.15)$ & 0.17 & $1.45(0.98,2.14)$ & 0.06 \\
\hline \multicolumn{7}{|c|}{ CTa $\left(\times 10^{4}\right)$, fourth vs. other quartiles } \\
\hline Sex, F & $2.37(0.89,6.26)$ & 0.08 & $1.99(0.70,5.67)$ & 0.20 & $1.37(0.65,2.91)$ & 0.41 \\
\hline \multicolumn{7}{|c|}{ CTv $\left(\times 10^{4}\right)$, first vs. other quartiles } \\
\hline Sex, F & $2.20(0.90,5.35)$ & 0.08 & $1.91(0.65,5.67)$ & 0.24 & $0.98(0.37,2.60)$ & 0.96 \\
\hline Duration, $\mathrm{y}$ & $0.81(0.69,0.96)$ & 0.01 & $0.86(0.72,1.01)$ & 0.07 & $0.96(0.84,1.10)$ & 0.58 \\
\hline
\end{tabular}

Bold values highlight statistically significant results.
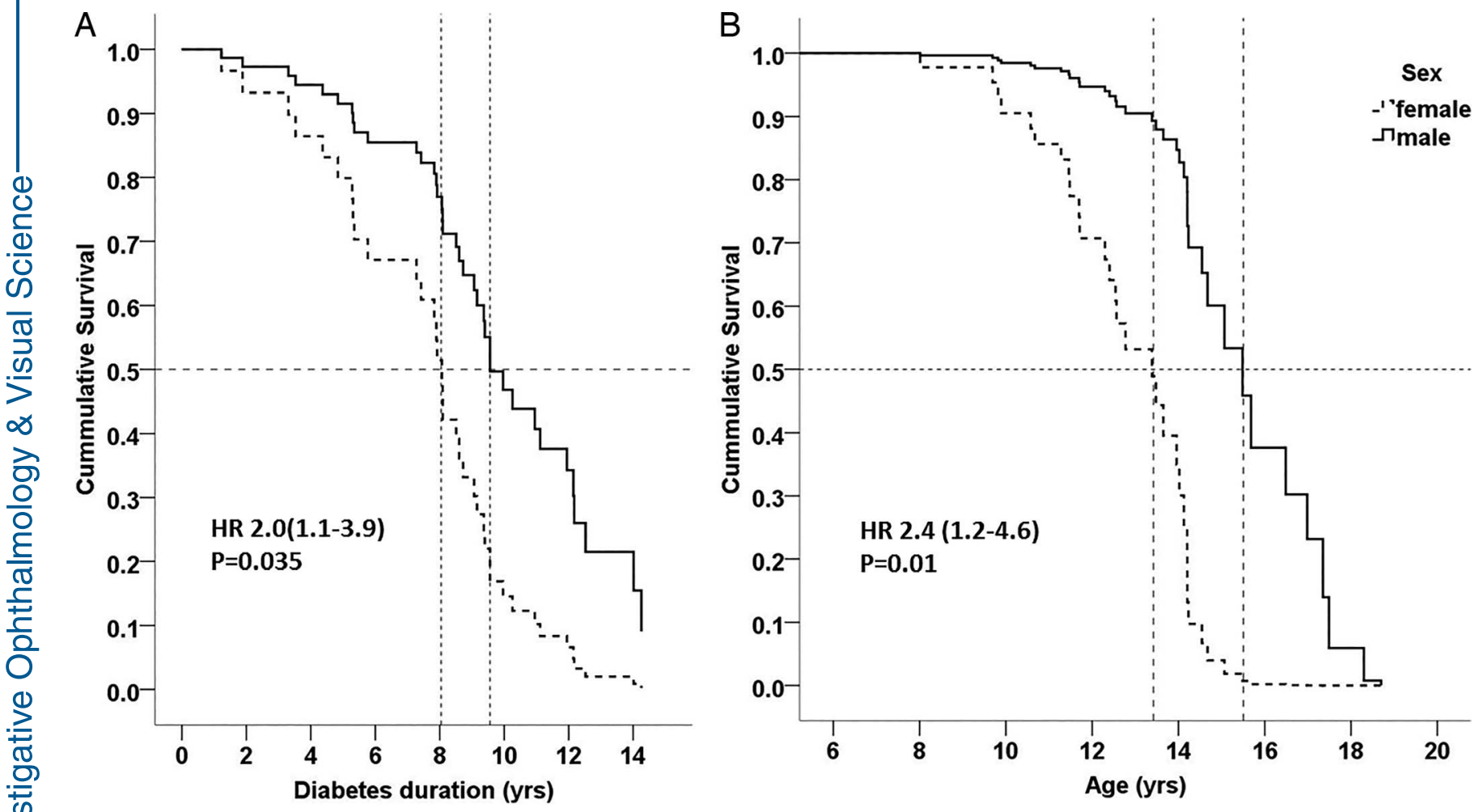

Figure. Multivariable Cox regression survival curves for risk of retinopathy by sex using (A) diabetes duration and (B) age as time-dependent variables. 
factor (IGF)-1/growth hormone (GH) axis and sex steroids levels,${ }^{1}$ may influence normal microvascular development or "maturation." IGF-1 plays an important role in the developing retinal vasculature and has a permissive role for VEGF action. ${ }^{30}$ Future studies of retinal vascular geometry, pubertal hormone levels and glycemic control/insulin therapy regimens will help to elucidate such mechanisms.

The strengths of this study include the detailed follow-up from pre- to post puberty, along with repeated RVG measures and complications assessments throughout the study period. Ideally pubertal hormones including IGF-1 and sex steroids would have been measured through the follow-up period but detailed pubertal assessments, including growth velocity, were performed at 3-month intervals by experienced pediatric endocrinologists. The sample size of this cohort may have been too small to detect causal associations between RVG and diabetes complications.

In conclusion, this is the first description of retinal vascular geometry from pre- to post puberty in adolescents with T1D. Sex dimorphism was evident with females having more complex arteriolar vascular network and simpler venular network than males, both patterns have previously been associated with greater risk of microvascular complications, however, the current study was unable to replicate these associations likely due to smaller sample size. Females were also at risk earlier incident retinopathy in this cohort. Retinal vascular geometry offers a unique opportunity to study and monitor the microvasculature precomplications and opens the possibility for an earlier screening paradigm in adolescents with diabetes in order to prevent clinical onset of microvascular complications. ${ }^{31}$ The significant differences in RVG observed from an early age suggest a role for heritability, which needs further exploration. This technology may assist in identifying high-risk groups in which early medical intervention may be of greatest benefit. Future studies including well characterized hormonal profiles through puberty and retinal vascular geometry will assist in furthering our understanding of the role of puberty (adverse or beneficial) in the development of microvascular complications.

\section{Acknowledgments}

Supported by grants from the National Health and Medical Research Council (NHMRC; Sydney, Australia), 475605 and the Juvenile Diabetes Research Foundation (JDRF; Sydney, Australia), 5-2008-274.

Disclosure: P. Benitez-Aguirre, None; M.E. Craig, None; H.G. Cass, None; C.J. Sugden, None; A.J. Jenkins, None; J.J. Wang, None; J. Cusumano, None; L.A.B. Hodgson, None; K. Lee, None; T.Y. Wong, None; K.C. Donaghue, None

\section{References}

1. Harvey JN. The influence of sex and puberty on the progression of diabetic nephropathy and retinopathy. Diabetologia. 2011;54:1943-1945.

2. Rogers DG, White NH, Shalwitz RA, Palmberg P, Smith ME, Santiago JV. The effect of puberty on the development of early diabetic microvascular disease in insulin-dependent diabetes. Diabetes Res Clin Practice. 1987;3:39-44.

3. Gallego PH, Craig ME, Hing S, Donaghue KC. Role of blood pressure in development of early retinopathy in adolescents with type 1 diabetes: prospective cohort study. BMJ. 2008; 337:a918.

4. Amin R, Widmer B, Prevost AT, et al. Risk of microalbuminuria and progression to macroalbuminuria in a cohort with childhood onset type 1 diabetes: prospective observational study. BMJ. 2008;336:697-704.
5. Xie XT, Liu Q, Wu J, Wakui M. Impact of cigarette smoking in type 2 diabetes development. Acta Pharmacol Sin. 2009;30: $784-787$.

6. Benitez-Aguirre P, Craig ME, Sasongko MB, et al. Retinal vascular geometry predicts incident retinopathy in young people with type 1 diabetes: a prospective cohort study from adolescence. Diabetes Care. 2011;34:1622-1627.

7. Benitez-Aguirre PZ, Sasongko MB, Craig ME, et al. Retinal vascular geometry predicts incident renal dysfunction in young people with type 1 diabetes. Diabetes Care. 2012;35: 599-604.

8. Donaghue KC, Fairchild JM, Chan A, et al. Diabetes microvascular complications in prepubertal children. $J$ Pediatr Endocrinol Metab. 1997;10:579-585.

9. Klein R, Klein BE, Moss SE, Davis MD, DeMets DL. The Wisconsin Epidemiologic Study of Diabetic Retinopathy. IX. Four-year incidence and progression of diabetic retinopathy when age at diagnosis is less than 30 years. Arch Ophthalmol. 1989; 107:237-243.

10. Benitez-Aguirre PZ, Sasongko BM, Craig M, et al. Retinal vascular geometry predicts incident renal dysfunction in young people with type 1 diabetes. Diabetes Care March. 2012;35:599-604.

11. Bonfanti R, Bognetti E, Meschi F, et al. Residual beta-cell function and spontaneous clinical remission in type 1 diabetes mellitus: the role of puberty. Acta Diabetol. 1998;35:91-95.

12. Malone JI. Ophthalmologic complications of insulin-dependent diabetes mellitus in children and adolescents. Pediatrician. 1983;12:194-198.

13. Mays D, Streisand R, Walker LR, Prokhorov AV, Tercyak KP. Cigarette smoking among adolescents with type 1 diabetes: strategies for behavioral prevention and intervention. $J$ Diabetes Complications. 2012;26:148-153.

14. Brink SJ. How to apply the experience from the diabetes control and complications trial to children and adolescents? Ann Med. 1997;29:425-438.

15. Witt N, Wong TY, Hughes AD, et al. Abnormalities of retinal microvascular structure and risk of mortality from ischemic heart disease and stroke. Hypertension. 2006;47:975-981.

16. Wong TY, Knudtson MD, Klein R, Klein BE, Meuer SM, Hubbard LD. Computer-assisted measurement of retinal vessel diameters in the Beaver Dam Eye Study: methodology, correlation between eyes, and effect of refractive errors. Ophthalmology. 2004;111:1183-1190.

17. Leung H, Wang JJ, Rochtchina E, Wong TY, Klein R, Mitchell P. Does hormone replacement therapy influence retinal microvascular caliber? Microvasc Res. 2004;67:48-54.

18. Nguyen TT, Wang JJ, Sharrett AR, et al. Relationship of retinal vascular caliber with diabetes and retinopathy: the MultiEthnic Study of Atherosclerosis (MESA). Diabetes Care. 2008; 31:544-549.

19. Kifley A, Wang JJ, Cugati S, Wong TY, Mitchell P. Retinal vascular caliber, diabetes, and retinopathy. Am J Ophthalmol. 2007; 143:1024-1026.

20. Kifley A, Wang JJ, Cugati S, Wong TY, Mitchell P. Retinal vascular caliber and the long-term risk of diabetes and impaired fasting glucose: the Blue Mountains Eye Study. Microcirculation. 2008;15:373-377.

21. Nguyen TT, Wang JJ, Wong TY, Nguyen TT, Wang JJ, Wong TY. Retinal vascular changes in pre-diabetes and prehypertension: new findings and their research and clinical implications. Diabetes Care. 2007;30:2708-2715.

22. Rydall AC, Rodin GM, Olmsted MP, Devenyi RG, Daneman D. Disordered eating behavior and microvascular complications in young women with insulin-dependent diabetes mellitus. $N$ Engl J Med. 1997;336:1849-1854.

23. Wang L, Wong TY, Sharrett AR, Klein R, Folsom AR, JeroschHerold M. Relationship between retinal arteriolar narrowing 
and myocardial perfusion: multi-ethnic study of atherosclerosis. Hypertension. 2008;51:119-126.

24. McGeechan K, Liew G, Macaskill P, et al. Meta-analysis: retinal vessel caliber and risk for coronary heart disease. Ann Intern Med. 2009;151:404-413.

25. Wong TY, Klein R, Sharrett AR, et al. Retinal arteriolar narrowing and risk of coronary heart disease in men and women. The Atherosclerosis Risk in Communities Study. JAMA. 2002;287:1153-1159.

26. Secrest AM, Becker DJ, Kelsey SF, LaPorte RE, Orchard TJ. Allcause mortality trends in a large population-based cohort with long-standing childhood-onset type 1 diabetes: the Allegheny County type 1 diabetes registry. Diabetes Care. 2010;33:25732579.

27. Mitchell P, Cheung N, de Haseth $\mathrm{K}$, et al. Blood pressure and retinal arteriolar narrowing in children. Hypertension. 2007; 49:1156-1162.
28. Liew G, Wang JJ, Klein R, et al. Birth weight is not related to risk of diabetic retinopathy in type 2 diabetes: the Atherosclerosis Risk in Communities Study. Curr Eye Res. 2008;33:193198.

29. Bulsara MK, Holman CD, Davis EA, Jones TW. The impact of a decade of changing treatment on rates of severe hypoglycemia in a population-based cohort of children with type 1 diabetes. Diabetes Care. 2004;27:2293-2298.

30. Sun T, Cao H, Xu L, Zhu B, Gu Q, Xu X. Insulin-like growth factor binding protein-related protein 1 mediates VEGFinduced proliferation, migration and tube formation of retinal endothelial cells. Curr Eye Res. 2011;36:341-349.

31. Ikram MK, Cheung CY, Lorenzi M, Klein R, Jones TL, Wong TY. Retinal vascular caliber as a biomarker for diabetes microvascular complications. Diabetes Care. 2013;36:750-759. 


\section{University Library}

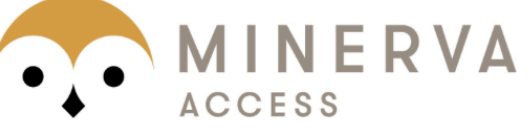

A gateway to Melbourne's research publications

Minerva Access is the Institutional Repository of The University of Melbourne

\section{Author/s:}

Benitez-Aguirre, P;Craig, ME;Cass, HG;Sugden, CJ;Jenkins, AJ;Wang, JJ;Cusumano, J;Hodgson, LAB;Lee, K;Wong, TY;Donaghue, KC

Title:

Sex Differences in Retinal Microvasculature Through Puberty In Type 1 Diabetes: Are Girls at Greater Risk of Diabetic Microvascular Complications?

Date:

2015-01-01

Citation:

Benitez-Aguirre, P., Craig, M. E., Cass, H. G., Sugden, C. J., Jenkins, A. J., Wang, J. J., Cusumano, J., Hodgson, L. A. B., Lee, K., Wong, T. Y. \& Donaghue, K. C. (2015). Sex Differences in Retinal Microvasculature Through Puberty In Type 1 Diabetes: Are Girls at Greater Risk of Diabetic Microvascular Complications?. INVESTIGATIVE OPHTHALMOLOGY \& VISUAL SCIENCE, 56 (1), pp.571-577. https://doi.org/10.1167/iovs.14-15147.

Persistent Link:

http://hdl.handle.net/11343/113660 